\title{
Horizons/Théâtre
}

Revue d'études théâtrales

10-11 | 2017

Genre et arts vivants

\section{Des costumiers aux costumières. Processus et conséquences d'une féminisation du secteur professionnel}

\section{Gaëlle Viémont}

\section{(2) OpenEdition \\ 1 Journals}

Édition électronique

URL : http://journals.openedition.org/ht/557

DOI : $10.4000 /$ ht.557

ISSN : 2678-5420

Éditeur

Presses universitaires de Bordeaux

\section{Édition imprimée}

Date de publication : 1 juillet 2017

Pagination : 202-218

ISSN : 2261-4591

\section{Référence électronique}

Gaëlle Viémont, «Des costumiers aux costumières. Processus et conséquences d'une féminisation du secteur professionnel », Horizons/Théâtre [En ligne], 10-11 | 2017, mis en ligne le 01 juillet 2018, consulté le 20 juillet 2019. URL : http://journals.openedition.org/ht/557 ; DOI : 10.4000/ht.557

\section{(9) $(\mathcal{\Theta \Theta}$}

La revue Horizons/Théâtre est mise à disposition selon les termes de la Licence Creative Commons Attribution - Pas d'Utilisation Commerciale - Pas de Modification 4.0 International. 


\section{GAËLLE VIÉMONT}

Gaëlle Viémont termine un doctorat à l'université de Strasbourg en arts du spectacle sous la direction de Geneviève Jolly. Ses recherches sont consacrées aux motifs historiques, économiques, socioculturels et genrés d'une méconnaissance des métiers du costume. Également titulaire d'un DMA costumier réalisateur et d'un diplôme de concepteur costume de l'ENSATT, elle exerce comme assistante et conceptrice costume depuis 2010. Elle est chargée d'enseignement à I'ENSATT et est enseignante contractuelle au DMA de La Martinière-Diderot. Elle a contribué à l'ouvrage collectif Faire œuvre sous le signe de la recherche publié sous la direction de Mireille Losco-Lena. Après avoir rédigé une douzaine de textes pour l'exposition Costumes de Légende du Musée des Tissus de Lyon (15 avril 2014 au 4 janvier 2015), elle est invitée par Delphine Pinasa à participer à l'exposition du CNCS « Artisans de la scène » (14 octobre 2017-11 mars 2018) et est l'autrice d'un chapitre du catalogue.

Mail : g.viemont@gmail.com

Résumé: : La terminologie de costumier est aujourd'hui vécue par les professionnels comme impropre à définir les différentes fonctions qu'ils exercent, alors même qu'ils demeurent attachés à ce vocable tant il situe leurs activités au sein des arts scéniques. Le problème langagier est si présent que le secteur costumier n'a de cesse de s'interroger sur les dénominations, ce que traduit l'apparition récente du vocable de " concepteur costume » qui, visant à différencier l'artiste des artisans, est aussi la marque d'une quête de valorisation. L'étude de l'origine du terme de « costumier » est indispensable à la compréhension de l'indétermination langagière qui frappe actuellement ce domaine artistique. Retracer la trajectoire de ce vocable conduit à opérer une lecture genrée de
I'histoire de la profession, tant il est clair que l'identité sexuée qui la caractérisait s'est inversée au cours du XIXe siècle. Elle est passée d'une activité définie comme masculine, à la fin $d u x v \|^{e}$ siècle, à une activité très majoritairement féminine au cours du $\mathrm{xx}^{\mathrm{e}}$ siècle. L'association entre les pratiques costumières et couturières a contribué à confondre, dans l'inconscient collectif, deux secteurs aussi corrélatifs que différenciés. Le tribut à payer par les professionnels de la discipline scénique est lourd en ce que leurs compétences sont tout autant essentialisées et minorées que celles des couturières.

Mots-CLÉs : costumier $\cdot$ ière, féminisation, art mineur, hiérarchie
Abstract: Nowadays, the terminology of costumier is considered by professionals as incorrect to define all their crafts, while they remain attached to the term as it situates them as members of the performing arts. The costume sector continues to question the pertinence of works designations as it is definitely considered as a language problem. In this context, the recent invention of an artistic terminology to identify clearly the costume designer in French, who is called "concepteur costume", constitutes an upgrading claim. The origin of the term costumier is still unknown despite its importance to understand the current indetermination of the specific vocabulary. Tracing the evolution of this terminology leads to a gendered interpretation of the profession's history, as far as it is obvious that its sexual identity was reversed trough time. It became a feminine sector during the twentieth century after being a male one till the end of the eighteenth century. The confusion between seamstresses and costumières participated in assimilating those corresponding, but separated, fields in the collective unconscious. The price to pay is heavy as it means naturalized skills and underestimated knowledge.

Kerwords: costumier-ière, feminization, minor art, hierarchy. 


\section{Des costumiers aux costumières. Processus et conséquences d'une féminisation du secteur professionnel}

L'art de créer les costumes de scène n'a jamais eu de nom dans la langue française et le terme de « costumier » est un hyperonyme loin de faire l'unanimité au sein du secteur d'activité. Rien n'est plus flou et obscur que ce métier, qui n'est pas un, mais multiple, et dont la terminologie est paradoxalement aussi impropre qu'explicite. Il représente une manière commode et généraliste de situer les praticien.ne.s d'un domaine professionnel atypique mais est incapable de rendre compte de la nébuleuse de métiers, aux expertises variables, qui collaborent à la création costumière. Il est enfin plus communément employé au féminin puisque $90 \%$ des costumiers sont des costumières ${ }^{1}$.

Le secteur professionnel est donc genré et le terme de « costumier » est plurivalent. Dans l'usage, le masculin désigne plus volontiers un concepteur costume quand « costumière » est confondu avec « couturière ${ }^{2} »$. Ces substantifs féminins sont fréquemment substitués l'un à l'autre, ce qui rend compte de l'imbrication historique entre le secteur de l'habillement et celui du costume de scène, mais illustre aussi le faible capital symbolique de la discipline costumière. En effet, on ne peut comprendre le secteur en dehors de l'industrie vestimentaire, des « régimes de genre ${ }^{3}$ » successifs qui caractérisent leur histoire et du stigmate social qui sanctionne les métiers de la couture, perçus comme des « métiers de toujours ${ }^{4} \gg$ pour les femmes. Or, ni les professions du costume ni celles du vêtement ne sont traditionnellement féminines et l'étude de leur genrisation permet d'analyser les motifs ségrégatifs d'une mésestimation actuelle de l'art costumier.

Parce que le langage « a une certaine responsabilité dans le maintien des discriminations ou des inégalités ${ }^{5} \gg$, il importe d'étudier l'inversion du genre $^{6} \mathrm{du}$ secteur costumier à travers la trajectoire et l'évolution sémantique du terme professionnel. S'intéresser en premier lieu au contexte d'apparition de celui-ci permettra de montrer qu'il définit à l'origine une discipline masculine érudite, issue du tailleur. Ensuite, nous verrons que l'inversion du genre n'illustre pas tant l'augmentation des travailleuses que le reflux des 
travailleurs dans un secteur qui tend à être assimilé à celui de la couture, et qui charrie des stéréotypes dépréciatifs. Enfin, nous montrerons, à l'aide d'extraits de paroles de professionnel.le.s, qui ont été recueillies dans le cadre de notre thèse de doctorat entre février 2013 et mars 2016, le poids du préjudice subi par un secteur artistique qui est toujours affilié symboliquement à un domaine traditionnellement déqualifié et mésestimé en raison de son caractère réputé féminin. Formée aux métiers de costumière-réalisatrice et de conceptrice costume, nous avons choisi de rechercher dans une lecture genrée de l'histoire les motifs d'une dévaluation de la pratique costumière en raison de constats effectués sur le terrain, lesquels ont été vérifiés et prolongés auprès des praticien-ne.s rencontrés.

\section{« Costumier » : une profession masculine}

Il est encore communément admis que les termes de « costumier, ière » sont attestés à partir de $1799^{7}$. Or, « costumier » apparaît pour la première fois en $1774^{8}$ - et uniquement au masculin - dans une lettre manifeste de Pierre-Nicolas Sarrazin ${ }^{9}$, publiée dans le Journal des Beaux-arts et des sciences ${ }^{10}$. Légitimé par sa qualité d'élève des Menus Plaisirs du Roi et de tailleur ordinaire de Monseigneur le Comte d'Artois, il se voit offrir une tribune publique pour développer ses théories professionnelles. Son argumentaire engagé vise la revalorisation du métier de tailleur en créant une « charge de nouvelle création qui ne se trouve pas dans l'État de France ${ }^{11} \gg$, profession qu' il baptise d'abord « Tailleur costumier ${ }^{12} \gg$ avant qu'elle ne devienne, sous sa plume, celle de $\ll \operatorname{costumier}^{13} \gg$. S'indignant du peu d'égard accordé à sa discipline, il s'ingénie à convaincre du bienfondé de considérer ce « nouvel » art comme relevant des arts libéraux, et de l'extraire du carcan infamant des arts mécaniques.

Le costumier est, selon lui, un homme éclairé, maitrisant l' histoire culturelle et sachant concevoir et couper un vestiaire théâtral en conformité avec les exigences historiques, dramaturgiques et esthétiques de la scène. L'art du costumier requiert le même degré d'érudition que les disciplines reconnues parmi les beaux-arts, puisqu'à l'instar des peintres et des sculpteurs, il « doit connaitre le Dessin, la Mythologie et l'Histoire ancienne et moderne, la Perspective, quelques principes d'Anatomie et de Géométrie pratique ${ }^{14} \gg$. Le tailleur costumier est donc un homme instruit dont la maîtrise des sciences listées est l'unique garantie d'un exercice professionnel de qualité. Ce dernier se démarque de la masse corporative par un ensemble de connaissances supervisant un ouvrage manuel et doit être « regardé comme un artiste 
savant dans son genre, et non pas comme un simple artisan, qui ne sait que prendre mesure et couper un habit ${ }^{15} \gg$.

La révolution professionnelle qu'entend mener Sarrazin passe par une distinction terminologique entre deux typologies antagonistes de praticiens, ceux qui se livrent, par habitude, à l'application stricte de protocoles techniques et ceux qui utilisent des connaissances variées dans une volonté perpétuelle de création et d'innovation. La figure élitaire que Sarrazin baptise est opposée aux ignorants manœuvres dénommés « Tailleurs routiniers ${ }^{16}$ », terminologie illustrant l'application d'automatismes figés. Pour asseoir ses doléances, Sarrazin a recours à une analyse se basant sur la mutation socioprofessionnelle de la peinture et de la sculpture durant le Grand Siècle ${ }^{17}$ et il crée le terme professionnel en le reliant explicitement à ces domaines.

« Costumier » est donc un néologisme élaboré à partir d'un vocable artistique importé d'Italie par Fréart de Chambray en 1662 dans son Idée de la perfection de la peinture. «Il Col tûme» est alors le « principe fondamental et universel pour toutes les parties de la Peinture ${ }^{18} \gg$, un outil conceptuel de jugement esthétique, intellectuel et moral de l'art pictural. Sa bonne observance garantit l'agent créateur $\mathrm{d}^{\prime} \ll$ absurdités ${ }^{19} \gg, \mathrm{d}^{\prime} \ll$ inepties et de lourdes fautes ${ }^{20} \gg$ qu'elles soient historiques, morales ou esthétiques, constatées chez des peintres manœuvres dépourvus de tout sentiment élevé de leur art. Appliqué au secteur des arts du spectacle, consécutivement à l'implantation « du concept de tableau pour désigner toute représentation ${ }^{21} \gg$, il conserve encore, un siècle plus tard, sa qualité conceptuelle, et « désigne en général tout ce qui dans les arts d'imitation sert à rendre l'imitation parfaite » et « doit régner plus impérieusement ${ }^{22}$ » au théâtre, notamment en ce qui concerne l'appareil visuel de la représentation, décors compris.

Prononcé durant près d'un siècle à l'italienne, « costume » s'est progressivement francisé avant de perdre les concepts qui lui étaient originellement attachés et est, en 1787, « fort en usage pour l'habillement des acteurs $^{23} \gg$. La restriction sémantique progressive de « costume » sous l'effet d'un « usage vulgaire $\gg$ a fait « perdre au mot sa signification primitive ${ }^{24} \gg$ en le rendant synonyme de « vêtement ». Parallèlement, « costumier » est entré dans le dictionnaire comme substantif masculin, semble-t-il en 1820, avec pour définition : «Tailleur qui fait, ou marchand qui vend ou loue des habits de théâtre, de bal, de mascarades ${ }^{25}$. $\gg$ Malgré les hautes vues de Sarrazin, il semble que son activité complémentaire et lucrative de loueur de costumes ait imprimé au terme un sens restreint, le privant de sa dimension artistique initiale. 
En effet, la fin des privilèges encourage vivement le commerce de la location de costumes qui répond aux besoins vestimentaires liés à la multiplication des salles de spectacle. S'il y en avait seulement une douzaine avant la Révolution française, on en dénombre une trentaine au moment des décrets napoléoniens de $1806-1807^{26}$. Il faut en outre ajouter les divertissements non théâtraux tels les mascarades et bals travestis qui, au moment du carnaval, génèrent un important chiffre d'affaires pour les loueurs de costumes. Perçus comme les médiateurs d'une consommation irraisonnable, ces derniers sont entachés d'une image défavorable. De l'avis de Nicolas Brazier, leurs commerces sont autant de « tristes réceptacle ${ }^{27} \gg$ où $\ll$ d'honnêtes ouvriers [vont] perdre leur temps et dépenser leur argent ${ }^{28} \gg$. Les costumiers sont décriés pour participer à la confusion des rangs par l'entremise du vêtement mais aussi pour leurs potentiels hauts gains commerciaux comme le remarque Louis Sébastien Mercier en affirmant que « chez une nation légère et folle, le masquier ou Costumier peut s'enrichir dans le cours d'un hiver ${ }^{29} \gg$.

La terminologie professionnelle s'est donc installée en deux endroits : celui du commerce pour théâtres et particuliers, et celui de l'artisanat tailleur appliqué à la scène. Force est de constater que le terme « costumier » n'a jamais été gratifié, dans le dictionnaire, de sa définition originelle, et son histoire socioprofessionnelle, sensiblement différente de ses principes théoriques, a imprimé au mot un sens ne correspondant pas à ses ambitions premières. Enfin, cette profession masculine était déjà victime d'une réputation défavorable avant qu'elle ne devienne une profession féminine, charriant bien d'autres stéréotypes négatifs.

\section{De « Costumier » à « costumière » : inversion du genre et « valence différentielle ${ }^{30}$ »}

Pierre-Nicolas Sarrazin, en tant que tailleur, relie les costumier.ière-s à un champ d'activité traditionnellement et symboliquement masculin. Le féminin de costumier ne semble pas employé pendant l'Ancien Régime, et n'apparaît dans la lexicographie que de manière tardive et surtout non systématique. Si le Grand dictionnaire universel du XIX ${ }^{e}$ siècle de $1869^{31}$ le référence, tel n'est pas le cas du Dictionnaire de l'Académie française de $1878^{32}$, pour lequel « costumier » demeure un substantif masculin. Si quelques tailleurs se définissent déjà comme costumiers dès 1791 avec Marillier au Théâtre de Monsieur et Simon à la Comédie-Française ${ }^{33}$, ils se multiplient au début du XIX ${ }^{\mathrm{e}}$ siècle $^{34}$. À l'inverse, il semble que les premières femmes à avoir été signalées comme costumières l'aient été de manière sporadique et isolée, en $1800^{35}, 1807^{36}$ et $1808^{37}$. 
Le dépouillement d'une dizaine d'almanachs et d'annuaires dramatiques depuis la fin du $\mathrm{XVIII}^{\mathrm{e}}$ siècle jusqu'au début du $\mathrm{Xx}^{\mathrm{e}}$ siècle montre que les premières costumières référencées sont encore des exceptions ${ }^{38}$. Il faut attendre les années 1830 pour constater un équilibre numérique entre le nombre des costumiers et des costumières répertorié.e.s, une mixité qu'on retrouve jusqu'à la Belle Époque, laquelle respecte la sacro-sainte séparation entre l'habillement masculin (tailleur) et l'habillement féminin (couture flou).

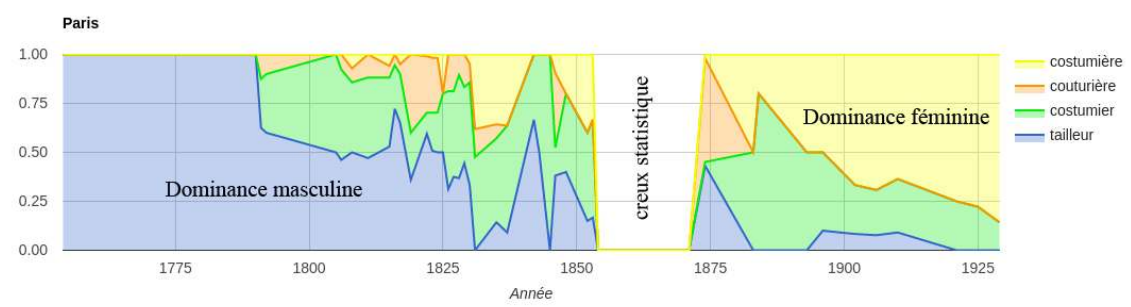

Proportion des tailleurs, couturières, costumiers et costumières référencée-s dans une dizaine d'almanachs et d'annuaires entre 1750 et 1925

En effet, les deux enquêtes statistiques pour la ville de Paris produites par la Chambre du Commerce en 1847-1848 et en 1860 rendent compte de cette répartition genrée. La première évalue à 195 ouvriers, costumiers et tailleurs et 219 ouvrières, costumières et couturières le personnel de 26 théâtres ${ }^{39}$, et la deuxième à « 157 costumiers et tailleurs » et $\ll 213$ couturières $^{40} \gg$ les membres des ateliers questionnés. L'enquête statistique sur l'industrie textile diligentée par Pierre du Maroussem en 1896 signale également des effectifs équilibrés pour les trois ateliers qu'il a interrogés ${ }^{41}$.

On constate la même tendance en ce qui concerne les loueur.se.s de costumes, dits « costumiers pour clients ${ }^{42} \gg$, qui embauchent 37 hommes et 47 femmes en $1847-1848^{43}, 25$ hommes et 37 femmes en $1860^{44}$. Pierre du Maroussem a recensé les effectifs d'un seul entrepreneur qui compte dans son « atelier patronal » trois hommes pour une femme mais déclare embaucher autant d'ouvriers sous-traitants de sexe féminin que de sexe masculin ${ }^{45}$. Or, il faut adjoindre à ces derniers leurs conjointes, dont le nombre est tu, qui sont signalées comme des aides non rémunérées. Cette « cécité statistique $^{46} \gg$ a été mise en lumière par plusieurs études féministes ${ }^{47}$ qui dénoncent l'« organisation de l'invisibilité du travail des femmes ${ }^{48}$ » par les recensements étatiques. Ce qui nous intéresse n'est donc pas tant le nombre 
exact de femmes dans les métiers du costume, mais le reflux des hommes qui apparaissent de moins en moins dans les répertoires, et ses causes, alors que l'emploi du féminin de « costumier » devient la règle au cours $\mathrm{du} \mathrm{Xx}^{\mathrm{e}}$ siècle et le substantif masculin acquiert une nouvelle aura artistique.

En effet, l'actuel prestige associé au substantif masculin n'est en rien comparable à l'imaginaire manufacturier charrié par le substantif féminin. Si les costumiers et les costumières sont au XIX ${ }^{\mathrm{e}}$ siècle les façonnier.ière.s d'un art pictural majoritairement signé par des hommes ${ }^{49}$, l'évolution des pratiques scéniques renforce une valence différentielle entre les deux substantifs. En effet, « costumier » supplante progressivement les appellations antérieures - dessinateur de costumes, décorateur et maquettiste - pour désigner le concepteur costume, formulation apparue au $\mathrm{XxI}^{\mathrm{e}}$ siècle. Cette évolution est sans doute corrélée à des mutations professionnelles sanctifiant la coupe historique ${ }^{50}$ des années 1970-1980 et par elle, des artisan·e.s devenu.e.s concepteur.rice.s, mais aussi à l'apparition de l'érudit vocable de 《scénographe » faisant péricliter celui de « décorateur ». Un chef d'atelier d'un opéra de région, que nous avons rencontré en 2014, témoigne de cette récente évolution du terme de « costumier » :

Le premier sens qui vient, c'est créateur de costumes. Avec le temps c'est devenu ainsi, mais autrefois, les anciennes couturières parlaient du décorateur pour le créateur de costumes, il ne faisait pas forcément du décor, mais c'était le décorateur $^{51}$.

À l'inverse, comme le montre cette même citation, le premier sens convoqué quand il est question de costumières est celui de « couturière » et l'apparition de « concepteur.trice costume » ou de « créateur.trice de costumes » est une tentative de clarification au sein de cette nébuleuse langagière. Enfin, relevons la partition genrée du discours ici énoncé : aux hommes la création et aux femmes l'exécution.

De fait, l'enquête que nous avons menée, et dont il sera question ciaprès, tend à indiquer que la minorité des hommes du secteur professionnel briguent les postes qui jouissent de la plus haute valeur sociale et, comme dans d'autres secteurs, « tirent souvent profit de leur "transgression" 52 ». Il existe donc, au sein de ce domaine professionnel fortement féminisé, dévalué par le patriarcat et l'économie libérale, une inégale distribution hiérarchique des postes qui, intrinsèquement liée au genre, se mesure à l'aune du capital symbolique différencié associé aux substantifs masculin et féminin que sont « costumier $»$ et $\ll$ costumière $»$. 


\section{Une occupation « naturellement » féminine, un métier de femmes}

En tant que discipline visant à vêtir le corps et utilisant les outils de l'artisanat vestimentaire, l'art costumier est donc rattaché aux domaines de l'habillement civil féminin et masculin. Comme le montrent les sources collectées, il y avait des tailleurs et des couturières, ainsi nommés, et opérant pour des artistes interprètes bien avant, et longtemps après, que le terme de « costumier.ière » ne les constitue en groupe singulier et spécifie leur champ d'exercice. Il faut donc relier les causes de l'inversion du genre ayant conduit à associer préférentiellement au terme de « costumier » une identité féminine à une histoire connexe, celle des métiers du vêtement. On peut en effet émettre l'hypothèse d'une corrélation entre l'assimilation de la couture à une occupation féminine et le reflux des costumiers dans le monde du spectacle.

La thèse publiée de Clare Haru Crowston ${ }^{53}$, qui étudie les motifs et les mécanismes d'une genrisation féminine du secteur de la couture, permet de comprendre comment le rapprochement idéologique entre les travaux d'aiguille et les femmes a pu se construire sous l'Ancien Régime. Elle montre que les professionnelles sont parvenues à faire reconnaître légalement leur pratique en empiétant considérablement sur le monopole couturier masculin, et en se positionnant, grâce à leur corporation fondée en 1675, en concurrentes directes et instituées des tailleurs. En tant que filles et femmes de tailleurs, les couturières dépendaient de l'autorité du patriarche dans l'exercice de leurs compétences. En tant que veuves, elles étaient soumises à la juridiction de la corporation de leur défunt époux, et dont les jurés étaient des hommes ${ }^{54}$. Elles étaient donc dans l'incapacité statutaire de faire entendre leur voix à égalité avec celle des maîtres tailleurs. Mais en tant que maîtresses couturières, appartenant à une corporation exclusivement féminine, les femmes étaient soustraites à la tutelle masculine, en ayant acquis, via la jurande, des droits équivalents à ceux prévalant dans n'importe quelle communauté de métier ${ }^{55}$.

D'âpres luttes les ont opposées aux tailleurs tout au long du XVIII ${ }^{\mathrm{e}}$ siècle, ces derniers étant peu enclins à voir leurs activités décliner tant l'institution des couturières en communauté de métier représentait pour eux « une perte économique, sociale, et culturelle ${ }^{56} \gg$. L'un des moyens rhétoriques principaux des couturières pour accroître leurs privilèges a consisté à défendre l'idée que la couture était une activité idoine de leur sexe. Elles ont de fait participé activement à la déqualification de leur pratique en la considérant comme une « compétence féminine biologiquement innée ${ }^{57}$ ». La corporation a en effet produit un discours stratégique valorisant des qualités morales 
et artisanales décrites comme intrinsèques au sexe féminin, permettant d'exclure les hommes, « aux mains grossières ${ }^{58}$ », et susceptibles de se rendre coupables de gestes déplacés et de regards libidineux. La stratégie fait fortune dans un contexte philosophique et politique propice à la séparation et la hiérarchisation des sphères masculines et féminines. La Pétition des femmes $d u$ Tiers-État au Roi, présentée le $1^{\text {er }}$ janvier 1789, reprenant les arguments des couturières, justifie ainsi la volonté d'évincer les hommes des métiers de l'aiguille dans une tactique émancipatrice :

Pour obvier à tant de maux, Sire, nous demandons : que les hommes ne puissent, sous aucun prétexte, exercer les métiers qui sont l'apanage des femmes, soit couturières, brodeuses, marchandes de modes, etc.; que l'on nous laisse au moins l'aiguille et le fuseau, nous nous engageons à ne manier jamais le compas et l'équerre ${ }^{59}$.

Or, si une telle stratégie a permis aux travailleuses du textile, et notamment aux marchandes de modes, de prospérer au début du $\mathrm{XIX}^{\mathrm{e}}$ siècle, une prolétarisation des ouvrières s'est renforcée avec la mise en place de la confection dès les années $1830-1840^{60}$. Les arguments biologiques sanctifiant une femme vertueuse, délicate et talentueuse sont progressivement devenus les moteurs d'une dépréciation symbolique et salariale. La confection a entraîné « une déqualification des couturières ${ }^{61}$ » qui sont devenues les travailleuses pauvres d'une économie de la sous-traitance, culottières et giletières de l'apparat masculin, monteuses et couseuses du vestiaire féminin, malgré des qualifications souvent nettement supérieures à celles attendues par leurs emplois. En parallèle, les grands couturiers, et parmi eux Charles Frederick Worth, ont renouvelé le secteur des marchandes de mode, et ont capitalisé sur une nouvelle identité d'artiste masculin dirigeant et s'opposant à la manufacture couturière féminine.

Les arguments d'une hiérarchie entre les disciplines du tailleur et du flou reposent sur une distinction entre la coupe à plat, réputée mathématique, complexe et périlleuse, et l'empirisme du moulage et la couture, fastidieuse et répétitive. Si les tailleurs se défendent au $\mathrm{XIX}^{\mathrm{e}}$ siècle en faisant valoir leur « génie créateur ${ }^{62}$ » porté par leur maîtrise des « proportions géométriques ${ }^{63}$ » et l'utilisation précoce du mètre, les couturières, aussi douées soient-elles, chiffonnent. Et pour cause, « la femme est capricieuse, bavarde, facile à distraire, et son travail ne peut se comparer à celui d'un homme ${ }^{64} \gg, y$ compris dans le secteur couturier. Comme le montre l'enquête de 1956 de Madeleine Guilbert et Viviane Isambert-Jamati, l'industrie emploie longuement un tel argument pour diminuer artificiellement la rémunération des ouvrières de 
sous-traitance au prétexte que sans la distraction de l'atelier, les couturières travaillent plus vite ${ }^{65}$. Or, non seulement les hommes et les femmes sont inégaux quant à la valeur pécuniaire de leurs « dons $»$ biologiques $-\ll$ la force physique est rétribuée ; mais pas la finesse des mains ${ }^{66} \gg-$ mais encore les couturières sont historiquement sous-payées en raison même de leur nature distraite et de leur fragile composition. Comme le constatait encore Yvonne Verdier en 1979, « c'est bien le fait de coudre qui est associé à la légèreté ${ }^{67}$ », et en tant que couseuses, les costumières n'échappent pas à ce stéréotype.

\section{La Costumière, la couturière, la ménagère}

L'histoire de la machine à coudre résume une politique capitaliste et patriarcale d'assimilation entre l'espace du foyer et les travaux couturiers. Conçue dans les années 1830 au service d'une concentration des ouvrier.ière.s dans des usines de confection pour un rendement majoré, elle est conspuée, en vain, par les tailleurs ${ }^{68}$. D'abord outil massif et viril d'une industrie mécanisée, elle devient, comme le montre Judith Coffin ${ }^{69}$, «l'un des premiers appareils ménagers ${ }^{70} \gg$ des intérieurs bourgeois du $\mathrm{XIX}^{\mathrm{e}}$ siècle. Lancée dans les années 1850 par Singer, « la familiale » est une machine à coudre aux lignes tortueuses et raffinées, destinée exclusivement aux femmes pour viser « l'accaparement d'un nouveau marché féminin ${ }^{71} \gg$. La confusion entre menus travaux domestiques et exercice professionnel à domicile sert le développement d'une industrie du prêt-à-porter qui est fondée sur l'emploi d'une main-d'œuvre féminine disséminée, mésestimée et sous-payée.

Un siècle plus tard, dans les années 1950, Singer s'adapte aux évolutions des mentalités et du marché, dans un climat de remise en cause de l'exploitation endémique des ouvrières à domicile, en présentant sa machine comme «le gardien des savoir-faire domestiques ${ }^{72} \gg$. Il n'est pas anodin que ces appareils s'éclaircissent à cette même époque, jusqu'à devenir blancs pour leur grande majorité, associant bel et bien leur fonction à l'espace sain et bien tenu du foyer. Les appellations de machines « familiale » et « industrielle » divisent toujours les machines à coudre en deux groupes distincts quand les premières sont les outils indispensables des costumier.ière.s indépendant.e.s et les secondes des travailleur.euse.s en ateliers. Somme toute, la confusion entre travaux ménagers et activité salariée perdure dans la taxinomie et dans la pratique costumière, liant précarité et espace privé, profession et espace public, comme peuvent en témoigner les professionnel.le.s actuels dans la série d'entretiens que nous avons réalisés.

Nous avons interrogé une trentaine de praticien.ne.s sur leurs parcours respectifs, leur vision du secteur professionnel, et les motifs probables de 
sa mésestimation et de sa sous-rémunération. Les plus âgés d'entre eux et d'entre elles ont commencé à exercer dans le milieu du $\mathrm{xx}^{\mathrm{e}}$ siècle quand les plus jeunes étaient à l'orée d'une carrière dans le domaine. L'idée était, audelà de consigner la parole peu audible des costumier.ière.s, de mesurer les effets durables d'une histoire collective dévalorisant les savoir-faire et les savoir-être ${ }^{73}$ associés au féminin. Il s'agissait d'analyser la manière dont ils et elles se présentaient, ce qu'ils et elles valorisaient parmi leurs compétences et ce qu' ils et elles estimaient subir, ou non, comme préjudice social en exerçant dans un domaine aussi fortement féminisé. Quels que soient leur tranche d'âge et leur sexe, ils et elles établissaient un lien majeur entre les femmes, la couture, l'espace domestique et la dévaluation des costumier.ière.s, ressentie collectivement. Ils et elles associaient les compétences des professionnel.le.s aux savoir-faire couturiers puisqu'ils constituent les fondements artisanaux de la pratique costumière. Et si les demandes du milieu du spectacle sont si diversifiées qu'elles exigent d'avoir recours à des procédés de plasticien·ne.s, l'aiguille et la machine à coudre demeurent les outils récurrents et principaux de l'art costumier, et les praticien.ne.s s'y réfèrent prioritairement pour décrire leurs métiers.

L'assimilation entre profession et espace domestique est ainsi signalée par une coupeuse qui estime que le défaut de reconnaissance de la discipline costumière tient au fait que la couture était une affaire de « ménagères des années 1950, avec l'idée qu'elles étaient chez elles et qu'elles ne faisaient $\operatorname{rien}^{74} \gg$. L'emploi de la terminologie de couturière peut également être perçu comme une mésestimation des compétences costumières :

Je n'aime pas dire que je suis couturière. [...] Pour moi, parler seulement de couturière est très réducteur. Je ne veux pas dire que c'est réducteur d'être couturière, mais c'est réducteur par rapport à ce qu'on fait tous les jours ${ }^{75}$.

Le vocable de couturière charrie avec lui un ensemble de représentations qui sont identifiées comme avilissantes et dévalorisantes. Savoir qu'elles ont été fabriquées par des décennies d'un dénigrement patriarcal et d'une exploitation outrancière bien favorable à la prospérité industrielle vestimentaire ne suffit pas à réhabiliter l'appellation. Et pour cause, elle qualifie un métier également abhorré par un nombre conséquent de femmes en ce qu'il leur a été imposé, auquel elles ont été conditionnées dès les premiers temps de leur scolarisation ${ }^{76}$. Cette réalité historique est énoncée par un concepteur costume, également scénographe, qui mesure pleinement l'écart de reconnaissance symbolique et salariale qu'il expérimente entre ses deux fonctions : 
C'est après coup que j'ai découvert que beaucoup de femmes de ma génération avaient des mères couturières qui leur avaient dit « Non, tu ne fais pas ce métier, c'est un mauvais métier, épuisant ». Ce qui est vrai. Et surtout, il est extrêmement mal payé, il n'est absolument pas valorisé $e^{77}$.

Une telle dévalorisation est résumée dans la perpétuation d'une expression normative, celle de « petites mains ». S'il s'agit d'un lieu commun, il est perçu par certain.e.s costumier.ière.s comme une désignation péjorative, et une nuisible banalisation de la hiérarchie genrée qui le fonde. Une coupeuse remarque en effet : « Les femmes sont les ouvrières, et les hommes sont les patrons ou contremaîtres. Elles étaient les "petites mains", on ne dit pas cela pour parler d'un homme ${ }^{78}$. $\gg$ Une cheffe habilleuse relie plus spécifiquement l'expression à la sphère domestique et au poids d'une telle appellation pour décrire les costumières :

Toutes les petites mains ne sont que des petites mains, cette expression est d'ailleurs très insultante. Est-ce à penser qu'il y en a des grandes? C'est joli, c'est gentil et très caressant, ces petites mains qui peuvent faire tous ces travaux à la maison en gardant les enfants ${ }^{79}$.

En effet, cette appellation de « petites mains » qualifie à l'origine le personnel féminin des ateliers de grande mesure situé au pied de la hiérarchie artisanale. L'expression désignait donc initialement des débutantes exerçant sous la responsabilité et l'autorité d'une seconde main et d'une première main $^{80}$. Il est finalement significatif que la postérité ait conservé une formulation rendant compte d'une position subalterne et mineure plutôt que celle de « première main », diminuant durablement l'art couturier et renvoyant directement au devoir de minutie, de discrétion et de docilité exigé des artisanes et des femmes.

C'est donc tout le poids de cette assignation et de cette minoration construite qui pèse sur les costumières actuelles. Ceci est immanquablement corrélé à l'identification de la couture en termes d'occupation féminine plutôt que d'activité professionnelle. Lourd est le tribut à payer par ce domaine artistique, dévalué en ce qu'il est attaché à la fois à une longue tradition ouvrière féminine, pétrie d'exploitation, et à la sphère privée, au lieu domestique et à l'inactivité supposée de la femme au foyer. Les discriminations se ressentent jusque dans le milieu universitaire tant il y est difficile d'envisager le domaine costumier selon les mêmes exigences critiques dont sont gratifiés d'autres secteurs des arts scéniques. Cette situation rend compte du défaut de prestige 
lié à la discipline et à son corollaire, l'absence de valorisation d'un tel objet d'étude, peu rémunérateur en termes de légitimité scientifique. Il y a donc profit à chercher les caractéristiques d'une minoration des métiers du costume dans une lecture genrée de l'histoire, mais aussi et surtout à utiliser cette grille d'analyse pour renouveler l'historiographie disciplinaire. On ne peut en effet faire l'économie d'une telle méthode sous peine de demeurer en surface des pratiques costumières et de faire disparaître sous l'étude des objets et des traces iconographiques les innombrables professionnel.le.s anonymes qui les ont laissés à la postérité.

\section{Notes}

1. Il s'agit du résultat d'une enquête de l'Observatoire prospectif des métiers du spectacle vivant, produite en septembre 2015 à partir d'indicateurs fournis par Audiens et Pôle emploi. Intitulée « Hommes et femmes dans le spectacle vivant. Regard sur la parité de l'emploi en 2013 ", elle a été pilotée par le CPNEF-SV (commission paritaire nationale emploi formation du spectacle vivant). L'enquête téléchargeable sur le site www. cpnefsv.org/donnees-statistiques a été consultée le 10 octobre 2015.

2. Les organigrammes de différentes structures culturelles consultables sur leurs sites (par exemple Opéra de Lyon, Angers-Nantes Opéra, Opéra national du Rhin) montrent que l'emploi de « couturière » perdure comme désignation professionnelle, et que le féminin est la règle même si l'équipe de couturières compte des hommes.

3. Lorena Parini, Le Système du genre. Introduction aux concepts et théories, Zürich, éditions Seismo, 2006, p. 35.

4. Sylvie Schweitzer, Les Femmes ont toujours travaillé. Une histoire du travail des femmes aux XIXe et XX siècles, Paris, éditions Odile Jacob, 2002, p. 12.

5. Muriel Plana, Théâtre et féminin. Identité, sexualité, politique, Dijon, Éditions universitaires de Dijon, 2012, p. 27.

6. Nous reprenons la formulation proposée par Yvonne Guichard-Claudic, Danièle Kergoat et Alain Vilbrod dans leur ouvrage L'Inversion du genre. Quand les métiers masculins se conjuguent au féminin... et réciproquement, Rennes, PUR, 2008.

7. Alain Rey (dir.), Dictionnaire historique de la langue française (1992), Paris, Le Robert, 2016, p. 571.

8. À ce sujet et en ce qui concerne une analyse historique et sémantique des termes de " costume " et de " costumier ", nous nous permettons de renvoyer à notre thèse de doctorat qui sera soutenue début 2018, Les Costumiers, ces orfèvres d'un art dramaturgique sans nom. Assises, enjeux et perspectives d'un secteur professionnel méconnu, sous la direction de G. Jolly, arts du spectacle, études théâtrales, ACCRA, Université de Strasbourg.

9. Artiste majeur pour l'histoire du costume et du spectacle en général, il a été oublié par la postérité. Nous avons mené de longues investigations à son sujet, elles constituent la première partie de notre thèse. Nous sommes en mesure d'affirmer qu'il est né en 1738 et qu'il est mort après 1803 . 
10. Pierre-Nicolas Sarrazin, « Lettre aux auteurs de ce journal, sur l'Art du Tailleur Costumier », dans Jean Louis Castilhon, Journal des Beaux-arts et des sciences, Paris, Moutard, article IX, troisième supplément, 1774, p. 93-106. Elle est suivie de deux autres lettres, publiées dans le même journal, en 1775 et 1776 .

11. Bertrand de La Tour, Réflexions morales, politiques, historiques et littéraires sur le théâtre, Avignon, Marc Chave, t. 17, 1775, p. 92.

12. Pierre-Nicolas Sarrazin, "Lettre aux auteurs de ce journal, sur l'Art du Tailleur Costumier ", op. cit., p. 96.

13. Pierre-Nicolas Sarrazin, "Projet d'établissement d'un Tailleur-Costumier ", dans Jean Louis Castilhon, Journal des sciences et des beaux-arts, dédié à Monseigneur le Comte d'Artois, Paris, Lacombe, tome 4, $1^{\text {er }}$ octobre 1776, p. 72-78.

14. Pierre-Nicolas Sarrazin, « Lettre aux auteurs de ce journal, sur l'Art du Tailleur Costumier », op. cit., p. 96.

15. Jacques Gautier Dagoty, Journal, dédié à Monsieur, frère du roi. Table générale des journaux anciens et modernes, $\mathrm{n}^{\circ}$ 3, Paris, Chez Demonville, 1776, tome 1, p. 368.

16. Pierre-Nicolas Sarrazin, "suite de la lettre de M. Sarrazin, sur l'Art du Tailleur Costumier ", Journal des Beaux-arts et des sciences, Paris, Moutard premier supplément, 1775 , p. 77.

17. Voir à ce propos les deux articles de Nathalie Heinich, «Arts et sciences à l'âge classique » et «La Perspective académique », Actes de la recherche en sciences sociales, [trimestrielle, depuis 1975], Paris, Le Seuil, vol. 66-67, mars 1987 et vol. 49, septembre 1983.

18. Roland Fréart de Chambray, Idée de la perfection de la peinture, Le Mans, Jacques Isambart, 1662, table, n. p.

19. Ibid., p. 55 .

20. Ibid., p. 57.

21. Emmanuelle, Hénin, Ut Pictura Theatrum. Théâtre et peinture de la Renaissance italienne au classicisme français, Genève, Droz, 2003, p. 608.

22. État Actuel de la Musique et des Trois Spectacles de Paris [annuel, 1767-1778], Paris, Vente, 1767, p. V et VII.

23. Jean-François Féraud, Dictionnaire critique de la langue française, Marseille, Mossy père et fils, t. I, 1787, p. 593.

24. Eustache Courtin, Encyclopédie moderne, ou Dictionnaire abrégé des sciences, des lettres et des arts, [1824-1832], Paris, Bureau de l'Encyclopédie, 26 vol., t. VIII, 1826, p. 543.

25. Jean-Charles Laveaux, Nouveau dictionnaire de la langue française : où l'on trouve le recueil de tous les mots de la langue usuelle, Paris, Deterville, 1820, p. 474.

26. Jean-Claude Yon, Une histoire du théâtre à Paris. De la Révolution à la Grande Guerre, Paris, Aubier Flammarion, 2012, p. 11 et 43.

27. Nicolas Brazier, Histoire des petits théatres de Paris depuis leur origine, Paris, Allardin, 1838, t. II, p. 157.

28. Ibid., p. 156.

29. Louis Sébastien Mercier, Néologie ou vocabulaire de mots nouveaux, à renouveler, et pris dans des acceptions nouvelles, Paris, Moussard et Maradan, 1801, p. 131.

30. On doit ce concept à Françoise Héritier, Masculin, Féminin. La pensée de la différence, Paris, Odile Jacob, 1996. 
31. Pierre Larousse, Grand dictionnaire universel du XIX siècle, Paris, administration du grand Dictionnaire universel, 1869, tome 5, p. 261.

32. Dictionnaire de l'Académie française, par l'Institut de France, $7^{\mathrm{e}}$ édition, Paris, FirminDidot et $\mathrm{C}^{\mathrm{ie}}$, tome 1, 1878, p. 411.

33. Almanach général de tous les spectacles de Paris, Paris, chez Froullé, 1791, p. 58 et p. 115.

34. Annuaire Dramatique ou étrennes théâtrales [1805-1822], Paris, Mme Cavanagh, 1805, p. 36, 154, 203, 217, 233 et p. 246.

35. Almanach des spectacles de Paris, ou calendrier historique et chronologique des théâtres [18001815], Paris, Duchesne, 1800, p. 108.

36. Sébastien Bottin, Jean de la Tynna, Almanach du commerce de Paris, Paris, bureau de l'almanach, 1807, p. 167.

37. Annuaire Dramatique ou étrennes théâtrales, op. cit., 1808, p. 142.

38. Outre les titres sus-cités, nous avons notamment consulté : Duverger et Arnaud, Indicateur Général des spectacles de Paris, des départements et des principales villes étrangères (1819-1823), Paris, bureau de l'Almanach du commerce ; Merville et Coupart, Almanach des spectacles (1822-1837), Paris, J.-N. Barda ; Almanach royal et national (1831-1847), Paris, chez A. Guyot et Scribe, Almanach des spectacles (1852-1853), Paris, chez M. Émile Brière, Albert Soubies, Almanach des spectacles (1874-1913), Paris, Libraire des Bibliophiles.

39. Natalis Rondot, Léon Say (dir.), Statistique de l'Industrie à Paris : résultat de l'enquête faite par la Chambre de commerce pour les années 1847-1848, Paris, Guillaumin, 1851, p. 943.

40. Émile Cottenet (dir.), Statistique de l'Industrie à Paris pour l'année 1860, Paris, Chambre de commerce, 1864, p. 1033.

41. Pierre Robert Planteau du Maroussem (dir.), La Petite Industrie : salaires et durée du travail. Le vêtement à Paris, demandé par l'Office du Travail, Paris, Imprimerie nationale, 1896, p. 682-685.

42. Ibid., p. 680 .

43. Natalis Rondot, Léon Say (dir.), Statistique de l'Industrie à Paris, op. cit., p. 248.

44. Émile Cottenet (dir.), Statistique de l'Industrie à Paris, op. cit., p. 264.

45. Pierre Robert Planteau du Maroussem (dir.), La Petite Industrie, op. cit., p. 680.

46. Sylvie Schweitzer, Les Femmes ont toujours travaillé, op. cit., p. 61.

47. Notamment Margaret Maruani, Monique Meron, Un siècle de travail des femmes en France, 1901-2011, Paris, La Découverte, 2012.

48. Sylvie Schweitzer, Les Femmes ont toujours travaillé, op. cit., p. 77.

49. Encore que cette idée pourrait être invalidée par des recherches spécifiques : les annuaires et almanachs consultés ne mentionnent que des hommes au titre de dessinateurs de costumes mais des femmes exerçaient bien comme loueuses de costumes, à l'instar de Delphine Baron, laquelle a joui d'un prestige certain en ce qui concerne la création de costumes de scène et de bals.

50. Jusqu'aux travaux de recherche sur la coupe et les patrons anciens amorcés au milieu $\mathrm{du} \mathrm{Xx}^{\mathrm{e}}$ siècle, menés par des chercheurs et des professionnels, les costumes historiques étaient obtenus à partir de patrons contemporains modifiés. La coupe historique exige de retrouver les lignes de construction révolues et de chercher dans la structure vesti- 
mentaire le style de chaque époque. Il s'agit-là de l'aboutissement artisanal du vérisme historique scénique théorisé à partir du XVIII ${ }^{\mathrm{e}}$ siècle.

51. Chef d'atelier, la quarantaine, entretien réalisé le 12 mars 2014.

52. Françoise Vouillot, Les Métiers ont-ils un sexe ?, Paris, Belin, 2014, p. 57.

53. Clare Haru Crowston, Fabricating Women, the Seamstresses of Old Regime France, 16751791, Londres, Duke University Press, 2001.

54. Voir Steven Laurence Kaplan, La Fin des corporations, Paris, Fayard, 2001.

55. Clare Haru Crowston, Fabricating Women, op. cit., p. 253. Notre traduction.

56. Ibid., p. 230.

57. Ibid., p. 11.

58. Jacques-Vincent Delacroix, Supplément au Mémoire à consulter des Six Corps, p. 3.

59. Pétition des femmes du Tiers-Etat au Roi, $1^{\text {er }}$ janvier 1789, [reproduction microforme], s. 1., Micro Graphix pour la BNF, « les archives de la Révolution française », 1992, p. 6.

60. Clare Haru Crowston, Fabricating Women, op. cit., p. 384.

61. Nancy L. Green, Du sentier à la $7^{\text {’̀me }}$ avenue. La confection et les immigrés Paris-New York 1880-1980, traduit de l'anglais par Pap Ndiaye, Paris, Éditions du Seuil, 1998, p. 110.

62. Augustin Canneva, "De la confection », dans Adolphe Goubaud (dir.), L'Élégant : journal des tailleurs, Paris, s. e., 20 juillet 1838, n 34, p. 269.

63. Bernard, "Patrons et confection ", ibid., 20 août 1837, n 23, p. 179.

64. Lucien de Vissec, Camille Frison, ouvrière de la couture, Paris, Librairie Plon, 1908, p. 18.

65. Madeleine Guilbert, Viviane Isambert-Jamati, Travail féminin et travail à domicile. Enquête sur le travail à domicile de la confection féminine dans la région parisienne, Paris, Centre national de la recherche scientifique, 1956, p. 152-153. $90 \%$ des travailleuses touchent, au moment de l'enquête, un salaire inférieur à ce qui est prévu par la loi.

66. Danièle Kergoat, Se battre, disent-elles..., Paris, La Dispute, 2012, p. 42.

67. Yvonne Verdier, "La couturière ", Façon de dire, façon de faire. La laveuse, la couturière, la cuisinière, Paris, Gallimard, 1979, p. 235.

68. Henriette Vanier, La Mode et ses métiers : frivolités et luttes des classes, 1830-1870, Paris, Armand Colin, 1960, p. 42.

69. Judith Coffin, « Naissance d'une consommation de masse en France, 1880-1914 », dans Louis Bergeron (dir.), La Révolution des aiguilles. Habiller les Français et les Américains, 19-20' siècles, Paris, Éditions de l'EHESS, 1996, p. 115-149.

70. Ibid., p. 141.

71. Ibid., p. 120.

72. Ibid., p. 143.

73. Si nous ne développons pas davantage cet aspect des compétences professionnelles des praticien·ne·s, nous nous permettons de renvoyer à nouveau à notre thèse de doctorat qui analyse les savoir-être costumiers sous l'angle du "souci de l'autre » de l'attention et du soin à l'aide des études féministes consacrées au care. Les travaux de Joan Tronto, de Patricia Paperman et de Sandra Laugier constituent des appuis théoriques intéressants pour envisager les comportements induits par le travail costumier et la place des agent·e.s dans la hiérarchie professionnelle, tels une posture d'écoute et d'attention vis-à-vis d'autrui, comme des acquis de l'expérience. Or, comme ces théoriciennes du 
care le montrent, ces savoir-être sont dévalués comme la marque de la faiblesse, de la soumission et du devoir féminin. Non seulement ceux-ci ne sont pas rémunérés comme des qualifications mais sont-ils encore les motifs insidieux d'une dévaluation supplémentaire, venant s'additionner à la mésestimation des savoir-faire costumiers.

74. Coupeuse, la quarantaine, entretien réalisé en octobre 2013.

75. Costumière réalisatrice, la vingtaine, entretien réalisé le 14 mars 2014.

76. Sylvie Schweitzer, Les Femmes ont toujours travaillé, op. cit., p. 219.

77. Concepteur costume et scénographe, la quarantaine, entretien réalisé le 25 mars 2014.

78. Costumière réalisatrice et coupeuse, la cinquantaine, entretien réalisé le 8 février 2013.

79. Cheffe habilleuse, la quarantaine, entretien réalisé le 24 mars 2014.

80. Lucien de Vissec, Camille Frison, op. cit., p. 11. 